\title{
EVALUATION OF PERIOPERATIVE HIGH-SENSITIVE CARDIAC TROPONIN I AS A PREDICTIVE BIOMARKER OF MAJOR ADVERSE CARDIOVASCULAR EVENTS AFTER NONCARDIAC SURGERY
}

\author{
Alejandro Millán-Figueroa ${ }^{\ddagger 1}$, Juan M. López-Navarro ${ }^{\ddagger 1}$, , IVÁn Pérez-DíaZ ${ }^{1,2 *}$, Jaime Galindo-Uribe ${ }^{3}$, \\ Blanca García-Martínez ${ }^{1}$, Sonia L. Del Villar-Velasco ${ }^{4}$, Tomás López-Gómez ${ }^{4}$, Zamar Zavaleta- \\ MartíneZ ${ }^{3}$, Eduardo J. Valladares-PÉreZ ${ }^{1}$, Hillary K. Osorio-Landa ${ }^{1}$, Andrea G. Nieto-Niño ${ }^{3}$, \\ Rodrigo F. Fernández-Pellón García ${ }^{3}$, and Reynerio Fagundo-Sierra ${ }^{4}$
}

\begin{abstract}
Departments of ${ }^{1}$ Internal Medicine and ${ }^{2}$ School of Medicine and Health Sciences, Instituto Tecnológico y de Estudios Superiores de Monterrey, Mexico City; ${ }^{3}$ Department of Cardiology and ${ }^{4}$ Central Laboratory, Instituto Nacional de Ciencias Médicas y Nutrición Salvador Zubirán, SSA, Mexico City, Mexico
\end{abstract}

₹These authors contributed equally to this study.

\begin{abstract}
Background: Various studies suggest that perioperative concentrations of high-sensitivity troponins are incremental and predictive factors of a major adverse cardiac event (MACE) and all-cause mortality. Objective: The objective of the study was to evaluate the predictive value of high-sensitivity cardiac troponin I (hs-cTnl) in the development of MACE and all-cause mortality, within 30-days and 1-year follow-up after noncardiac surgery. Methods: In this prospective cohort study, we included men $\geq 45$ years and women $\geq 55$ years with $\geq 2$ cardiovascular risk factors and undergoing intermediate or high-risk noncardiac surgery. Demographic and clinical information was collected from clinical charts. We measured baseline hs-cTnl $24 \mathrm{~h}$ before surgery, and its post-operative concentration $24 \mathrm{~h}$ after surgery. Results: In the entire sample, 8 patients ( $8.6 \%$ ) developed MACE at 30-days follow-up (4 deaths), 12 (12.9\%) within the $1^{\text {st }}$ year (7 deaths), and 17 (18.2\%) after complete post-surgical follow-up (10 deaths). We observed higher baseline and post-operative concentrations in patients who presented MACE (12 pg/ml vs. $3.5 \mathrm{pg} / \mathrm{ml} ; \mathrm{p}=0.001$ and $18.3 \mathrm{pg} / \mathrm{ml}$ vs. $5.45 \mathrm{pg} / \mathrm{ml} ; \mathrm{p}=0.009$, respectively). The hazard ratios (HRs) calculated by Cox regression analysis between the hs-cTnl baseline concentration and the post-operative development of MACE at 30-days and 1-year were 5.70 (95\% confidence interval [Cl], 1.10-29.40) with hs-cTnl > $6.2 \mathrm{pg} / \mathrm{ml}$ and 12.86 (95\% Cl, 1.42-116.34) with hs-cTnl > $3.3 \mathrm{pg} / \mathrm{ml}$, respectively. The estimated post-operative HR death risk at $1-y e a r$ was 14.43 (95\% Cl, 1.37-151.61) with hs-cTnl > $4.5 \mathrm{pg} / \mathrm{ml}$. Conclusions: Pre-operative hs-cTnl was an independent predictive risk factor for MACE at 30-days and 1-year after noncardiac surgery and for all-cause mortality at 1-year after noncardiac surgery. (REV INVEST CLIN. 2020;72(2):110-8)
\end{abstract}

Key words: Cardiac troponins. Noncardiac surgery. Major adverse cardiac event. Mortality.

*Corresponding author:

Iván Pérez-Díaz

E-mail: ivan.endocrino@gmail.com
Received for publication: 29-12-2018

Approved for publication: 24-01-2019

DOI: $10.24875 / R I C .19002888$

0034-8376 / (c) 2019 Revista de Investigación Clínica. Published by Permanyer. This is an open access article under the CC BY-NC-ND license (http://creativecommons.org/licenses/by-nc-nd/4.0/). 


\section{INTRODUCTION}

Cardiac troponins ( $\mathrm{cTn}$ ) are structural proteins of the cardiac myocyte contractile apparatus and the preferred biomarker in the detection of a myocardial lesion $^{1-3}$. According to the Fourth Universal Definition of Myocardial Infarction, the term acute myocardial infarction should be used when cTn values rise and/ or fall with at least 1 value above the $99^{\text {th }}$ percentile upper reference limit and at least one of the following: symptoms of myocardial ischemia, new ischemic electrocardiographic changes, development of pathological Q waves, imaging proof of new myocardial injury, or the presence of an intracoronary thrombus by angiography or autopsy ${ }^{4}$. In patients subjected to a surgical procedure, perioperative stress has been reported to precipitate the development of major adverse cardiac events (MACEs) ${ }^{5}$. New high-sensitivity assays can detect very low circulating troponin levels in the general population. These concentrations correlate with the prevalence of associated cardiovascular risk factors, metabolic abnormalities, and/or cardiac dysfunction ${ }^{6}$. The elevation of high-sensitivity troponin in the perioperative period has recently been suggested to be of further prognostic value in the detection of cardiovascular complications ${ }^{5}$. The aim of this study was to evaluate the clinical usefulness of high-sensitivity cTn I (hs-cTnl) in the prediction of MACEs and mortality in patients with established cardiovascular disease or cardiovascular risk factors undergoing noncardiac surgical interventions.

\section{METHODS}

We conducted a prospective and longitudinal cohort study in a tertiary care hospital between August 2014 and October 2017. The study was approved by the Institute's Ethics Committee, and a signed clinical consent was obtained from each patient according to the international recommendations in the clinical search.

\section{Patients and hs-cTnl measurements}

We identified males $\geq 45$ years of age and females $\geq$ 55 years of age who, according to the stratification of cardiac risk in noncardiac surgical procedures of the American Heart Association (AHA) and the American
College of Cardiology $(A C C)^{7}$, would undergo intermediate- or high-risk noncardiac surgery. We collected demographic and clinical data from the patients' clinical charts and only included in the study patients who fulfilled at least two of the following cardiovascular risk factors: a history of ischemic heart disease (IHD), cerebral vascular disease (CVD), or heart failure (HF); insulin-dependent diabetes mellitus; dyslipidemia; renal failure with serum creatinine values $\geq 2$ $\mathrm{mg} / \mathrm{dL}$ or in replacement therapy; smoking; and hypertension. All patients with an acute coronary syndrome within $24 \mathrm{~h}$ before surgery were excluded from the study. Patients were stratified according to the revised cardiac risk index (RCRI) for pre-operative (PreOp) risk, and the Gupta risk index 8,9 .

PreOp hs-cTnl values were determined $24 \mathrm{~h}$ before surgery, and a second measurement was obtained 24 h after surgery (PostOp). The ARCHITECT STAT High Sensitive Troponin-I assay was used (B3P253, Abbott Laboratories, Chicago, IL), which has a variation coefficient $\leq 10 \%$ in the $99^{\text {th }}$ percentile and measurable concentrations in at least $50 \%$ of healthy individuals below the $99^{\text {th }}$ percentile. The upper reference limit, corresponding to the value in the $99^{\text {th }}$ percentile in a healthy reference population, is $26.2 \mathrm{pg} / \mathrm{ml}$ according to the assay's insert ( $15.6 \mathrm{ng} / \mathrm{L}$ in females and 34.2 $\mathrm{ng} / \mathrm{L}$ in males).

\section{Definitions and outcomes}

A MACE was defined as the development of an acute coronary syndrome, cardiac arrest, congestive HF requiring hospitalization, percutaneous or surgical coronary revascularization, CVD, peripheral arterial thrombosis, and death due to any cause. The primary outcome was the development of MACE and all-cause mortality within the $1^{\text {st }}$ month. The secondary outcome was considered to be the development of MACE and all-cause mortality after a 1-year follow-up and during the overall follow-up. Follow-up was conducted by telephone and review of the patients' medical charts.

\section{Statistical analysis}

We evaluated data normality with the KolmogorovSmirnov test. Variables with a normal distribution are expressed as means ( $m$ ) and standard deviations (SD), while those without a normal distribution are 
expressed as medians (med) and interquartile ranges. We compared quantitative variables with a normal distribution with Student's t-test, quantitative variables with a non-normal distribution with MannWhitney U-test, and categorical variables with Pearson's Chi-square test $\left(X^{2}\right)$. For the comparison of the pre- and post-levels of hs-cTnl, a t-test for independent samples or Wilcoxon signed-rank test was used according to the distribution. We divided the hs-cTnl measurements into quartiles and compared the events that occurred in each quartile with a $X^{2}$ test. We calculated the hazard ratios (HRs) with $95 \%$ confidence intervals ( $\mathrm{Cls}$ ) for the development of MACE and mortality at 30 days, 1 year, and throughout overall follow-up. We conducted a multivariate analysis by Cox regression after adjusting for the patients' comorbidities to evaluate the persistence of the detected associations.

ROC curves were created for each outcome to evaluate the test's performance. We established two cutoff points for the different outcomes: the first cutoff point, which was obtained using a multivariate model, represents the PreOp hs-cTnl concentration from which we observed an increased risk of MACE or death; and the second cutoff point, which was obtained according to the highest value of the Youden Index, represents the PreOp hs-cTnl concentration with the best performance. To obtain an internal validation of our study, we carried out a bootstrapping analysis to evaluate the distribution of the difference in probability of MACE for different PreOp hs-cTnl cutoffs. We also calculated the area under the curve (AUC) of the risk indices in the RCRI and Gupta, to compare them with the PreOp hs-cTnl concentration AUC following the method recommended by DeLong et al. ${ }^{10}$.

Survival was evaluated by Kaplan-Meier curves and log-rank test. Finally, to evaluate the added predictive ability of the PreOp hs-cTnl to the Gupta risk index, we calculated the integrated discrimination improvement (IDI) and the net reclassification improvement (NRI). All hypothesis tests were twotailed and $p<0.05$ was considered statistically significant. Statistical analysis was performed with SPSS Statistics 23 software (IBM Corporation, Armonk, NY), XLSTAT 2017.1 (Addinsoft SARL, New York, NY), and MedCalc Software 18.2.1 (MedCalc, Ostend, Belgium).

\section{RESULTS}

We included 93 patients $(n=93)$ in the study, of which 50 patients $(53.7 \%)$ were male. The mean age was 67.1 years (SD \pm 8.9 years). Median overall follow-up after surgery was 602 days. In the entire sample, 17 patients (18.2\%) developed MACE distributed as follows: a total of 8 patients ( $8.6 \%$ ) developed MACE at 30-days follow-up, 12 patients (12.9\%) during the $1^{\text {st }}$ year, and 17 patients (18.2\%) within the overall follow-up. Among patients who developed MACE, we observed 4 deaths in the $1^{\text {st }}$ month, 7 deaths at 1-year follow-up, and 10 deaths in the overall follow-up. No differences were found in sex, age, prevalence of comorbidities, and RCRI between patients with and without MACE, either between alive or dead patients (Table 1 ).

In the total sample, the hs-cTnl concentration increased postoperatively when compared with the PreOp measurement $(3.8 \mathrm{pg} / \mathrm{ml}$ vs. $6.3 \mathrm{pg} / \mathrm{ml}$; $\mathrm{p}<$ 0.000). In patients with MACE, the PreOp and the post-operative concentrations were greater than in those who did not develop MACE (PreOp hs-cTnl: 12 $\mathrm{pg} / \mathrm{ml}$ vs. $3.5 \mathrm{pg} / \mathrm{ml}, \mathrm{p}=0.001$; and PreOp hs-cTnl: $18.3 \mathrm{pg} / \mathrm{ml}$ vs. $5.45 \mathrm{pg} / \mathrm{ml}, \mathrm{p}=0.009$, respectively; Table 2).

After dividing the PreOp and post-operative concentrations and their difference into quartiles, we observed that in the last quartile of PreOp hs-cTnl (7.06-772.4 pg/ml), a greater incidence of MACE occurred during the overall and 1-year follow-up points when compared with the rest of the inferior quartiles $(p=0.001$ and $p=0.006$, respectively). A similar tendency of MACE incidence was observed at the $1^{\text {st }}$ month. As to the PreOp concentration, we only found a greater incidence of MACE in the last quartile (16.71-750.5 pg/ml) during the overall follow-up when compared with the rest of the inferior quartiles $(p=0.005)$. No significant differences were observed in all other comparisons (Fig. 1).

Table 3 shows the PreOp hs-cTnl cutoff points with their respective HRs $(95 \% \mathrm{Cl})$ from which there is an increased risk for the different outcomes obtained by Cox regression analysis (Table S1 shows the bivariate analysis). These association measures were adjusted for age, gender, and the presence of comorbidities, 
Table 1. General characteristics of the population

\begin{tabular}{|c|c|c|c|c|c|}
\hline \multirow[t]{2}{*}{ Variables (\%) } & \multirow[t]{2}{*}{ Total Sample } & \multirow[t]{2}{*}{ No MACE group } & \multirow[t]{2}{*}{ MACE group } & \multirow{2}{*}{$\frac{\text { MACE group }}{\text { Alive }}$} & \multirow{2}{*}{$\frac{\text { MACE group }}{\text { Death }}$} \\
\hline & & & & & \\
\hline Patients & $93(100)$ & $76(81.7)$ & $17(18.3)$ & $7(41.2)$ & $10(58.8)$ \\
\hline Males & $50(53.76)$ & $41(53.95)$ & $9(52.5)$ & $3(42.86)$ & $6(60.0)$ \\
\hline Age in years $( \pm S D)$ & $67.1(8.9)$ & $67.4(8.5)$ & $66.7(10.8)$ & $69.7(11.0)$ & $64.1(10.8)$ \\
\hline IHD & $16(17.2)$ & $13(17.10)$ & $3(17.64)$ & $1(14.28)$ & $2(20.0)$ \\
\hline $\mathrm{CHF}$ & $13(13.97)$ & $9(11.84)$ & $4(23.52)$ & $2(28.57)$ & $2(20.0)$ \\
\hline Stroke & $11(11.82)$ & $7(9.21)$ & $4(23.52)$ & $2(28.57)$ & $2(20.0)$ \\
\hline $\mathrm{HT}$ & 65 (69.89) & $53(69.73)$ & $12(70.58)$ & $5(71.42)$ & $7(70.0)$ \\
\hline T2DM & $27(29.03)$ & $21(27.63)$ & $6(35.29)$ & $4(57.14)$ & $2(20.0)$ \\
\hline CKD & $30(32.25)$ & $24(31.57)$ & $6(35.29)$ & $3(42.85)$ & $2(20.0)$ \\
\hline Smoking & $52(55.91)$ & $44(57.89)$ & $8(47.05)$ & $2(28.57)$ & $6(60.0)$ \\
\hline Chol >200 mg/dL & $14(15.05)$ & $12(15.78)$ & $2(11.76)$ & $1(14.28)$ & $1(10.0)$ \\
\hline $\mathrm{C}-\mathrm{HDL}<40 \mathrm{mg} / \mathrm{dL}$ & $31(33.33)$ & $25(32.89)$ & $6(35.29)$ & $2(28.57)$ & $4(40.0)$ \\
\hline Malignancy & $39(41.93)$ & $32(42.10)$ & $7(41.17)$ & $1(14.28)$ & $6(60.0)$ \\
\hline \multicolumn{6}{|l|}{ RCRI } \\
\hline Lee I & $41(44.08)$ & $34(44.73)$ & $7(41.17)$ & $2(28.57)$ & $5(50.0)$ \\
\hline Lee II & $26(27.95)$ & $22(28.94)$ & $4(23.52)$ & $2(28.57)$ & $2(20.0)$ \\
\hline Lee III & $18(19.35)$ & $15(19.73)$ & $3(17.64)$ & $1(14.28)$ & $2(20.0)$ \\
\hline Lee IV & $8(8.60)$ & $5(6.57)$ & $3(17.64)$ & $2(28.57)$ & $1(10.0)$ \\
\hline
\end{tabular}

MACE: major adverse cardiac event; SD: standard deviation; IHD: ischemic heart disease; CHF: chronic heart failure; HT: hypertension; T2DM: type 2 diabetes mellitus; CKD: chronic kidney disease; Chol: total cholesterol; C-HDL: high-density lipoprotein cholesterol; RCRI: Revised Cardiac Risk Index.

Categorical variables were compared employing the Pearson's Chi-square test and quantitative variables the Student's t-test.

In all cases, the No MACE Group was compared to the MACE group, as well as the Alive versus Death in the MACE group. No statistically significant differences were observed between groups.

Table 2. Behavior of pre-operative and post-operative hs-cTnl concentration

\begin{tabular}{|c|c|c|c|}
\hline hs-cTnl & Min-Max (pg/ml) & Med in $\mathrm{pg} / \mathrm{ml}$ (IQR) & $p$ value \\
\hline \multicolumn{4}{|c|}{ Total sample $n=93$} \\
\hline PreOp & $0.00-772.40$ & $3.8(2.3-7.25)$ & $<0.000^{\times}$ \\
\hline PostOp & $0.00-750.50$ & $6.3(3.3-17.1)$ & \\
\hline \multicolumn{4}{|c|}{ No MACE Group n=76 } \\
\hline PreOp & $0.00-52.60$ & $3.5(2.2-5.97)$ & $\mathrm{a}<0.000^{\times}$ \\
\hline \multirow[t]{2}{*}{ PostOp } & $0.00-750.50$ & $5.45(3.3-10.82)$ & ${ }^{\mathrm{b}} 0.001^{\dagger}$ \\
\hline & & & ${ }^{c} 0.009^{\dagger}$ \\
\hline \multicolumn{4}{|c|}{ MACE Group n=17 } \\
\hline PreOp & $1.40-772.40$ & $12(14.15-15.80)$ & $0.094^{x}$ \\
\hline PostOp & $2.10-568.60$ & $18.3(4.25-90.2)$ & \\
\hline
\end{tabular}

hs-cTnl: high-sensitivity cardiac troponin I; IQR: interquartile range; MACE: major adverse cardiac event; Max: maximum; Med: median; Min: minimum; PostOp: post-operative; PreOp: pre-operative.

aComparison between PreOp and PostOp hs-cTnl in No MACE group. ${ }^{\mathrm{b} C o m p a r i s o n}$ of PreOp hs-cTnl between No MACE and MACE Groups.

${ }^{c}$ Comparison of PostOp hs-cTnl between No MACE and MACE Groups. ${ }^{\times}$Wilcoxon signed-rank test. ${ }^{\dagger}$ Mann-Whitney U-test. 
Table 3. Multivariate analysis (Cox regression) of pre-operative hs-cTnl cutoff points from which an increased risk of MACE or death was observed

\begin{tabular}{lccc}
\hline $\begin{array}{l}\text { PreOp hs-cTnl } \\
\text { HR }\end{array}$ & At 30-days & At 1 year & Overall follow-up \\
\hline PreOp hs-cTnl $(\mathrm{pg} / \mathrm{ml})$ & $>6.2$ & $>3.3$ & $>3.4$ \\
HR MACE $(95 \% \mathrm{Cl})$ & $5.70(1.10-29.40)$ & $12.86(1.42-116.34)$ & $4.13(1.05-16.13)$ \\
PreOp hs-cTnl $(\mathrm{pg} / \mathrm{ml})$ & $\mathrm{NS}$ & $>4.5$ & $>4.2$ \\
HR death $(95 \% \mathrm{Cl})$ & & $14.43(1.37-151.61)$ & $4.40(0.83-23.16)$ \\
\hline
\end{tabular}

hs-cTnl: high-sensitivity cardiac troponin I; MACE: major adverse cardiac event; PreOp: pre-operative; HR: hazard ratio; Cl: confidence interval; NS: nonsignificant.

The multivariate model was adjusted for age, gender, history of ischemic heart disease, chronic heart failure, hypertension, Type 2 diabetes mellitus, chronic kidney disease, Revised Cardiac Risk Index, serum creatinine, and glomerular filtration rate.

Figure 1. Incidence of MACE at 30-days, at 1-year, and throughout overall follow-up, in each pre-operative and post-operative hs-cTnl quartile and changes in each.

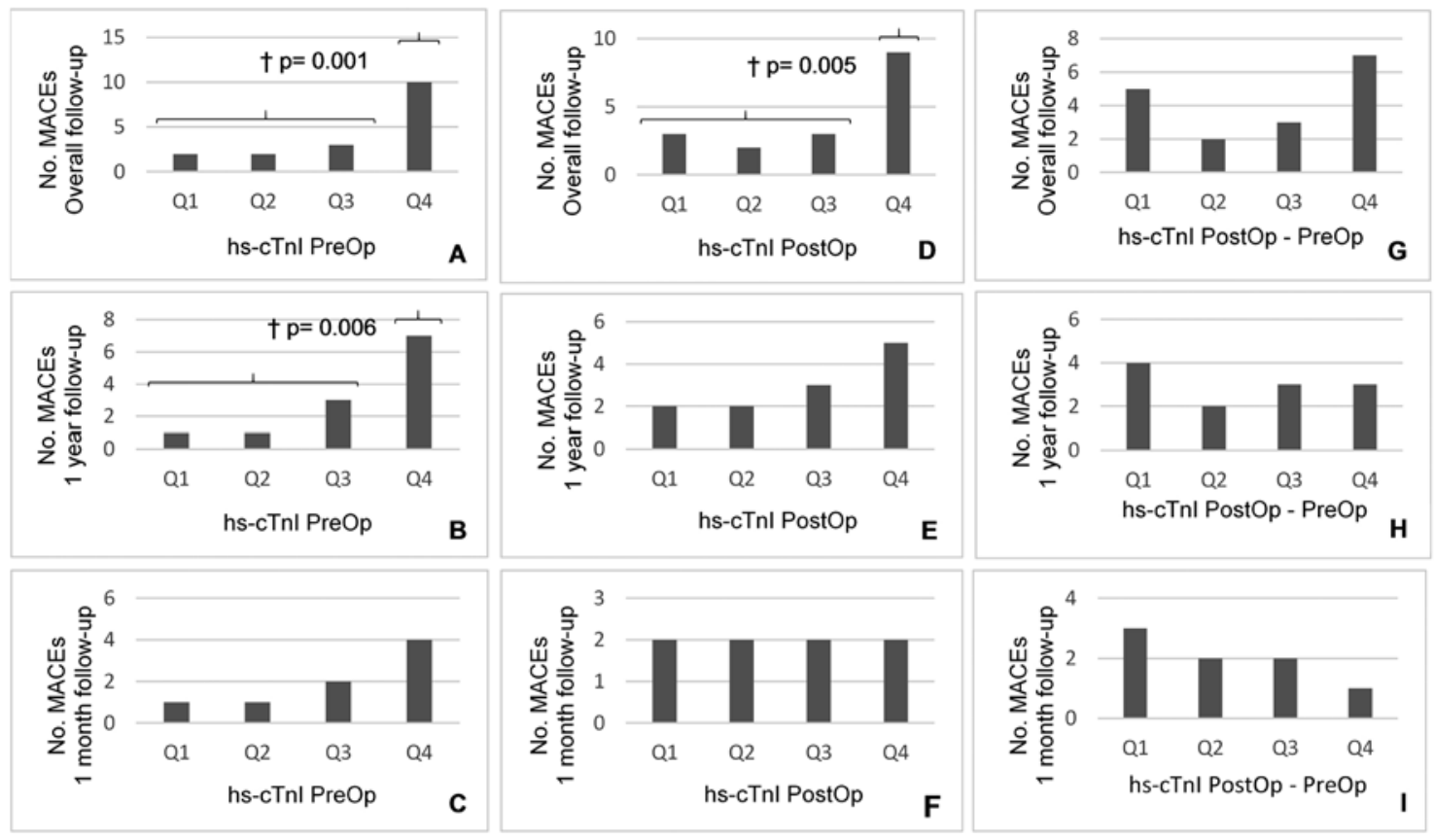

hs-cTnl: high-sensitivity cardiac troponin I, MACEs: major adverse cardiac events, PostOp: post-operative, PreOp: pre-operative, Q: quartile. PreOp hs-cTnl (pg/ml) quartile limits: Q1 (0-2.3), Q2 (2.31-3.8), Q3 (3.81-7.05), Q4 (7.06-772.4). PostOp (pg/ml) quartile limits: Q1 (0-3.3), Q2 (3.31-6.25), Q3 (6.26-16.7), Q4 (16.71-750.5). PostOp limits of the hs-cTnl difference quartiles and PreOp concentration: Q1 (-2103.8-0.1), Q2 (0.11-1.3), Q3 (1.31-6.2), Q4 (6.21-747). 'Pearson's Chi-square test (only significant comparisons are shown).

including RCRI. We must emphasize that we did not detect a significantly increased mortality risk at the 30-days follow-up point with the PreOp hs-cTnl concentration in bivariate and multivariate analyses or for mortality at the overall follow-up in multivariate analysis. The bootstrapping analysis shows a normal distribution when analyzing the following cutoff points: $3.3 \mathrm{pg} / \mathrm{ml}(95 \% \mathrm{Cl}, 0.0301-0.3191), 4.5 \mathrm{pg} / \mathrm{ml}(95 \%$ $\mathrm{Cl}, 0.0716-0.4196)$, and $6.2 \mathrm{pg} / \mathrm{ml}(95 \% \mathrm{Cl}, 0.1229-$ 0.5101 ; Fig. S1).
In the ROC analysis, we observed a higher PreOp hscTnl AUC for MACE and mortality incidence at 1-year and overall follow-up in comparison with the 30-days follow-up AUC. Table S2 shows the measures of accuracy of the identified PreOp hs-cTnl cutoff points. By comparing the AUC of the PreOp hs-cTnl concentration, the Gupta risk index, and the RCRI for the different outcomes, we found greater discriminatory ability of the PreOp hs-cTnl than the RCRI for the incidence of MACE during the overall follow-up (AUC 
Figure 2. Kaplan-Meier survival analysis for the development of MACE or death according to the pre-operative hs-cTnl.

MACE

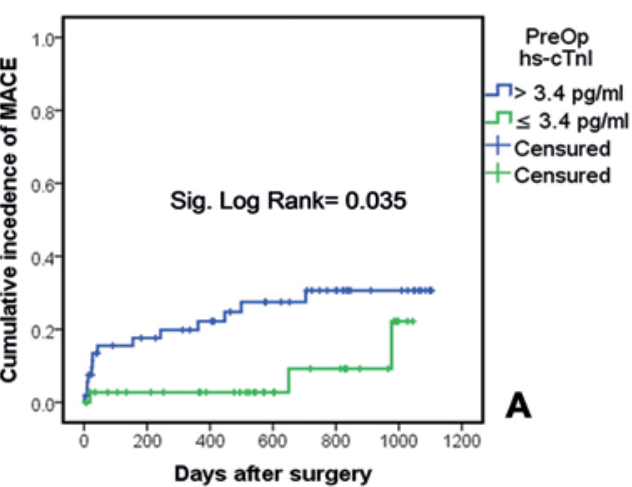

At 1-year follow-up

Overall follow-up
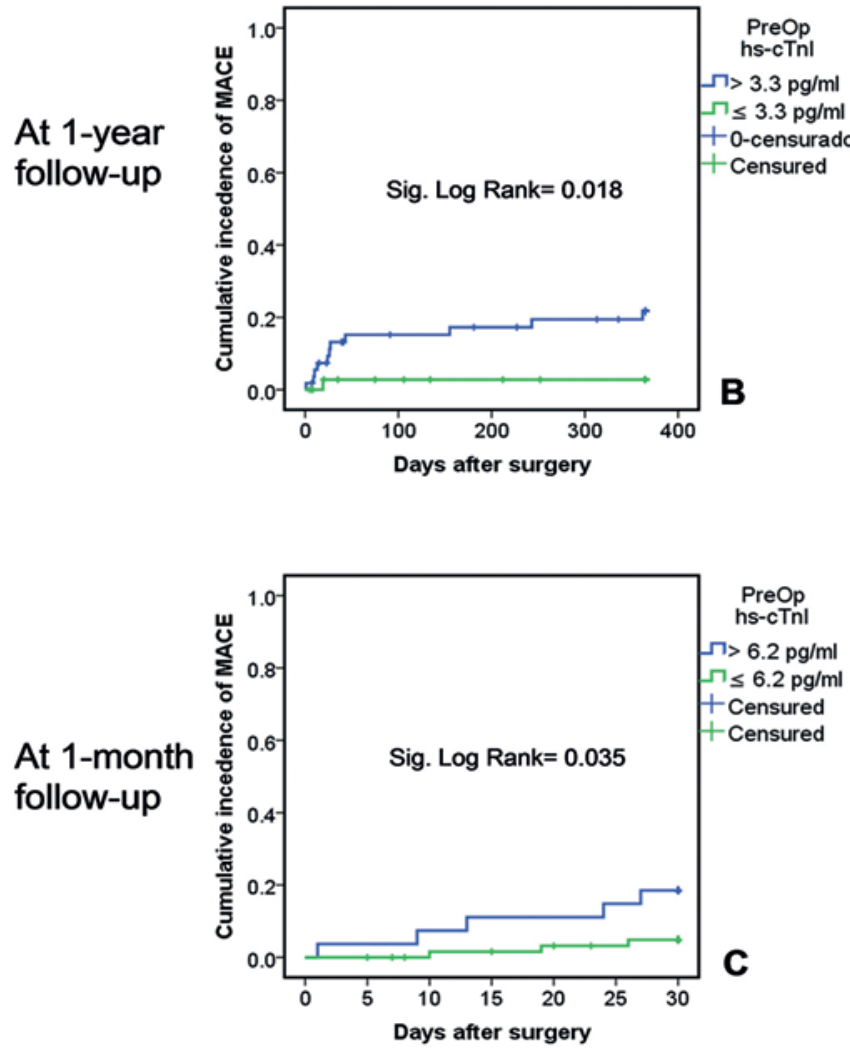
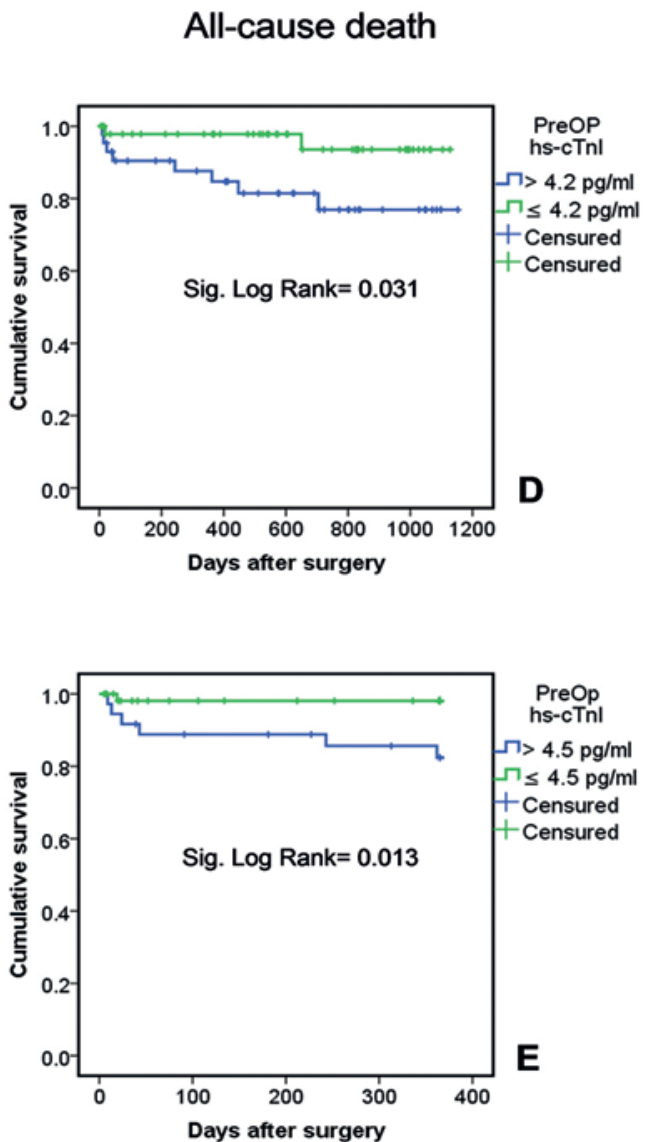

hs-cTnl: high-sensitivity cardiac troponin I; MACE: major adverse cardiac event; PreOp: pre-operative.

0.750, [95\% Cl, 0.649-0.834] vs. AUC 0.546, [95\% $\mathrm{Cl}, 0.439-0.650] ; \mathrm{p}=0.0387$ ), and also of the Gupta risk index compared with the RCRI (AUC 0.720, [95\% $\mathrm{Cl}, 0.616-0.808]$ vs. AUC 0.546, [95\% Cl, 0.4390.650 ]; $p=0.0387$ ). All other comparisons were not significant (Table S3). Using Kaplan-Meier survival analysis, we found that patients with a PreOp hs-cTnl concentration above the established cutoff points had a greater incidence of MACE and decreased survival (log rank $=0.005)$ (Figs. 2 and S2).

We determined by NRI analysis $(p<0.05)$ that when using all cutoff points identified in the Cox regression, the PreOp hs-cTnl and Gupta risk index combination 
improved the classification of patients with high risk $(\geq 1 \%)$ or low risk of developing MACE in comparison with the Gupta risk index (33.55\% for MACE at 30days; $8.70 \%$ for MACE and $16.72 \%$ for death at 1-year; and $7.70 \%$ for MACE and $11.55 \%$ for death within the overall follow-up). Furthermore, by IDI analysis we observed an integrated positive difference in Youden's indices with the new model (PreOp hs-cTnl and Gupta risk index combination) in comparison with the Gupta risk index for prediction of MACE at 1-year (IDI 15.56; 95\% Cl, 3.24-27.88; $\mathrm{p}=0.014$ ) and within the overall follow-up (IDI $3.73 ; 95 \% \mathrm{Cl}, 0.92-6.55$; $p=0.010$ ) (Table S4).

\section{DISCUSSION}

More than 200 million noncardiac surgical procedures are performed in adults every year throughout the world ${ }^{11}$, thus increasing the incidence of cardiac death by $0.5 \%-1.5 \%$ and the incidence of MACE by $2 \%$ to $3.5 \%^{12}$. Since cardiovascular disease remains the main cause of death worldwide ${ }^{13}$, the prevention of cardiovascular complications is a current challenge to health systems.

Our findings suggest that elevated PreOp hs-cTnl concentrations in patients with cardiovascular risk factors are independently associated to the development of MACE and death. Borges et al. reported that a peak level of PostOp hs-cTnl $>40 \mathrm{pg} / \mathrm{ml}$ was associated to decreased survival and decreased event-free survival by day $30^{14}$. Likewise, a greater increase in the PreOp and PostOp $\Delta$ hs-cTn is associated with a greater incidence of the analyzed outcomes ${ }^{5,15-18}$. Nevertheless, our results show that the PreOp concentration is superior and more clearly associated with the development of MACE in the short and long terms than the post-operative measurement or $\Delta$ hs-cTn, with which we detected no relationship whatsoever.

Some studies have found decreased survival and greater incidence of cardiac events when the hs-cTnT PreOp concentration is elevated, using the $99^{\text {th }}$ percentile cutoff point in acute events $(14 \mathrm{pg} / \mathrm{ml})^{15,19,20}$ or even a greater value $(\geq 17.8 \mathrm{pg} / \mathrm{ml})^{5}$. In our study, PreOp hs-cTnl concentrations remarkably below the $99^{\text {th }}$ percentile represented an increased risk for the evaluated outcomes. Perhaps both troponins ( $T$ and I) behave in a similar manner since the VISION study reported that a peak measurement of PostOp hscTnT of $5-14 \mathrm{pg} / \mathrm{ml}$ increases the risk of death 3.73 -fold at 30 days when compared with a concentration $<5 \mathrm{pg} / \mathrm{ml}$ (16). Likewise, some studies, as well as some meta-analyses conducted in the general population or the aging population that have not undergone a surgical procedure, have suggested that elevated baseline cTn T or I, below the 99th percentile, is significantly associated with an increased risk of cardiovascular death and all-cause mortality ${ }^{1,2,6,21-24}$.

Our study revealed that the risks of MACE and of death begin to increase in accordance with the PreOp hs-cTnl concentration as follows: patients with a value $>6.2 \mathrm{pg} / \mathrm{ml}$ have a 5 -fold risk of developing MACE in the $1^{\text {st }}$ month after surgery, a concentration $>3.3 \mathrm{pg} / \mathrm{ml}$ leads to a 12 -fold risk of developing MACE within a year after surgery, and even a value $>4.5 \mathrm{pg} / \mathrm{ml}$ leads to a 14 -fold increased risk of dying from any cause in the $1^{\text {st }}$ year after surgery. However, the cutoff points with the best performance are as follows: $>6.8 \mathrm{pg} / \mathrm{ml}$ for MACE at 1 month, $>7.3 \mathrm{pg} / \mathrm{ml}$ for death at 1 month, and > $6.0 \mathrm{pg} / \mathrm{ml}$ for MACE, and death during the $1^{\text {st }}$ year. These latter values correlate best with those proposed for the general population $(>6 \mathrm{pg} / \mathrm{ml})^{6}$. We must emphasize that all the mentioned cutoff points have a high negative predictive value ( $>92 \%$ ) for all outcomes, similar to the results reported with hs-cTnTs; their main use would, therefore, be the identification of patients who, in spite of harboring cardiovascular risk factors, do not require further PreOp evaluation.

The ACC/AHA 2014 perioperative cardiovascular evaluation clinical guidelines recommend that in patients at high risk of developing MACE $(\geq 1 \%)$ and poor or unknown functional capacity $(<4$ METs or metabolic equivalents), a stress test is suggested if it could potentially impact decision making or perioperative care, with the required subsequent approach and management according to the results ${ }^{25}$. Our study suggests that adding a PreOp hs-cTnl measurement to the Gupta risk index improves the classification of patients at low- or high-risk of developing MACE in the short- and long-term postoperative period (30 days and 1 year, respectively) and at low- or high-risk of long-term post-operative death, as well as increases the predictive ability of 
MACE in the long-term post-operative period. Further studies with a greater number of patients are necessary to confirm the added value of PreOp hs-cTnl to the Gupta perioperative risk index, as well as to determine the test's impact and cost-effectiveness.

One of our study's strengths is that, to the best of our knowledge, this is the first analysis evaluating the risk of different outcomes with PreOp hs-cTnl concentrations below the $99^{\text {th }}$ percentile; it is also the first to compare the predictive ability of the Gupta risk index when adding the PreOp hs-cTnl value. One of its limitations is the fact that it was conducted in a single center with a small number of patients. Another limitation is that several types of surgeries were included. A strikingly greater proportion of patients with cancer was observed among those who died compared with those who did not, but this difference was not statistically significant $(p=0.059)$, and the multivariate model was adjusted for malignancy diagnosis. Nevertheless, due to the influence of cancer deaths, it is necessary to consider the results with caution.

In conclusion, patients with cardiovascular risk factors, a PreOp hs-cTnl value above our proposed cutoff points increases the risk of postoperatively developing MACE in the short term and of postoperatively developing MACE or death in the long term. The PreOp hs-cTnl has discrimination similar to the Gupta risk index for the development of MACE and death. However, it seems that the combination of the Gupta risk index and the PreOp hs-cTnl further improve the correct classification of low- or high-risk patients and the predictive ability of MACE.

\section{ACKNOWLEDGMENTS}

We would like to thank Abbott Laboratories (Abbott Laboratories, Chicago, IL) for providing the in vitro assays used in the study. The authors would also like to thank Dr. Pablo A. Peña from Microanalítica, Mexico, for his helpful technical support.

\section{SUPPLEMENTARY DATA}

Supplementary data are available at Revista de Investigación Clínica online (www.clinicalandtranslationalinvestigation.com). These data are provided by the corresponding author and published online for the benefit of the reader. The contents of supplementary data are the sole responsibility of the authors.

\section{REFERENCES}

1. van der Linden N, Klinkenberg LJ, Bekers O, et al. Prognostic value of basal high-sensitive cardiac troponin levels on mortality in the general population: a meta-analysis. Medicine (Baltimore). 2016;95:e5703.

2. Willeit $P$, Welsh $P$, Evans JD, et al. High-sensitivity cardiac troponin concentration and risk of first-ever cardiovascular outcomes in 154,052 participants. J Am Coll Cardiol. 2017; 70:558-68.

3. Li P, Enea NS, Zuk R, et al. Performance characteristics of a high-sensitivity cardiac troponin assay using plasma and whole blood samples. Clin Biochem. 2017;50:1249-52.

4. Thygesen K, Alpert JS, Jaffe AS, et al. Fourth universal definition of myocardial infarction (2018). Circulation. 2018;138: e618-51.

5. Gillmann HJ, Meinders A, Grohennig A, et al. Perioperative levels and changes of high-sensitivity troponin $\mathrm{T}$ are associated with cardiovascular events in vascular surgery patients. Crit Care Med. 2014;42:1498-506.

6. Blankenberg S, Salomaa V, Makarova N, et al. Troponin I and cardiovascular risk prediction in the general population: the biomarCaRE consortium. Eur Heart J. 2016;37:2428-37.

7. Eagle KA, Berger PB, Calkins $\mathrm{H}$, et al. ACC/AHA guideline update for perioperative cardiovascular evaluation for noncardiac surgery-executive summary: a report of the American college of cardiology/American heart association task force on practice guidelines (Committee to update the 1996 guidelines on perioperative cardiovascular evaluation for noncardiac surgery). J Am Coll Cardiol. 2002;39:542-53.

8. Lee TH, Marcantonio ER, Mangione CM, et al. Derivation and prospective validation of a simple index for prediction of cardiac risk of major noncardiac surgery. Circulation. 1999; 100: 1043-9

9. Gupta PK, Gupta H, Sundaram A, et al. Development and validation of a risk calculator for prediction of cardiac risk after surgery. Circulation. 2011;124:381-7.

10. DeLong ER, DeLong DM, Clarke-Pearson DL. Comparing the areas under two or more correlated receiver operating characteristic curves: a nonparametric approach. Biometrics. 1988; 44: $837-45$

11. Devereaux PJ, Sessler DI. Cardiac complications in patients undergoing major noncardiac surgery. $N$ Engl J Med. 2015; 373 : 2258-69.

12. Mureddu GF. Current multivariate risk scores in patients undergoing non-cardiac surgery. Monaldi Arch Chest Dis. 2017; $87: 848$.

13. World Health Organization. The Top 10 Causes of Death; 2018. Available from: http://www.who.int/mediacentre/factsheets/ fs310/en/. [Last accessed on 2018 Oct 20].

14. Borges FK, Furtado MV, Rossini AP, et al. Clinical use of ultrasensitive cardiac troponin I assay in intermediate-and high-risk surgery patients. Dis Markers. 2013;35:945-53.

15. Nagele P, Brown F, Gage BF, et al. High-sensitivity cardiac troponin $\mathrm{T}$ in prediction and diagnosis of myocardial infarction and long-term mortality after noncardiac surgery. Am Heart J. 2013;166:325-320

16. Writing Committee for the VISION Study Investigators, Devereaux PJ, Biccard BM, et al. Association of postoperative highsensitivity troponin levels with myocardial injury and 30-day mortality among patients undergoing noncardiac surgery. JAMA. 2017;317:1642-51.

17. Puelacher C, Lurati Buse G, Seeberger D, et al. Perioperative myocardial injury after noncardiac surgery: incidence, mortality, and characterization. Circulation. 2018;137:1221-32.

18. Marston N, Sandoval Y, Zakharova M, et al. Troponin elevations following vascular surgery in patients without preoperative myocardial ischemia. South Med J. 2013;106:612-7.

19. Weber M, Luchner A, Seeberger $M$, et al. Incremental value of high-sensitive troponin $\mathrm{T}$ in addition to the revised cardiac index 
for peri-operative risk stratification in non-cardiac surgery. Eur Heart J. 2013;34:853-62.

20. Noordzij PG, van Geffen O, Dijkstra IM, et al. High-sensitive cardiac troponin $\mathrm{T}$ measurements in prediction of non-cardiac complications after major abdominal surgery. Br J Anaesth. 2015;114:909-18.

21. Eggers KM, Venge $P$, Lindahl B, Lind L. Cardiac troponin I levels measured with a high-sensitive assay increase over time and are strong predictors of mortality in an elderly population. J Am Coll Cardiol. 2013;61:1906-13.

22. Eggers KM, Al-Shakarchi J, Berglund L, et al. High-sensitive cardiac troponin $\mathrm{T}$ and its relations to cardiovascular risk factors, morbidity, and mortality in elderly men. Am Heart J. 2013;166:541-8.
23. Zeller $T$, Tunstall-Pedoe $H$, Saarela $O$, et al. High population prevalence of cardiac troponin I measured by a high-sensitivity assay and cardiovascular risk estimation: the MORGAM biomarker project scottish cohort. Eur Heart J. 2014;35:271-81.

24. Lewis JR, Lim WH, Wong G, et al. Association between highsensitivity cardiac troponin I and cardiac events in elderly women. J Am Heart Assoc. 2017;6:e004174.

25. Fleisher LA, Fleischmann KE, Auerbach AD, et al. 2014 ACC/ AHA guideline on perioperative cardiovascular evaluation and management of patients undergoing noncardiac surgery: a report of the American college of cardiology/American heart association task force on practice guidelines. J Am Coll Cardiol. 2014;64:e77-137.

\section{Erratum}

In the article by Bello-Chavolla OY and Aguilar-Salinas CA. "Factors Influencing Achievement of Low-Density Lipoprotein Cholesterol Goals in Mexico: The International Cholesterol Management Practice Study", published In Rev Invest Clin. 2019;71(6):408-416, doi: 10.24875/RIC.19003156, it was inadvertently omitted the name of Julieta de la Luz (from Sanofi, Mexico) as second author, on behalf of the Mexico's ICLPS group conformed by César Gonzalo Calvo Vargas (University of Guadalajara, Guadalajara Jal., Mexico), Edmundo Bayram Ilamas (Fundación Cardiovascular, Aguascalientes, Ags, Mexico), Esperanza Martinez Abundis (University of Guadalajara, Guadalajara Jal., Mexico), Gerardo Andres Baez Vargas (private practice, Mexico City, Mexico), Pedro Mendoza Martinez (Hospital Angeles Lindavista, Mexico City, Mexico), Rodrigo Navarrete Valencia (private practice, Mexico City, Mexico), Bernardo Emilio Valenzuela Salazar (private practice, Chihuahua, Chih., Mexico ), Francisco Javier Robledo Gutierrez (private Practice, Mexico City, Mexico), Alfredo Nacoud Askar (Faculty of Medicine, Universidad Autónoma de Nuevo Leon, Monterrey, N.L., Mexico), Carlos Alberto Aguilar Salinas (Instituto Nacional de Ciencias Médicas y Nutrición SZ, Mexico City, Mexico), Sergio Zuñiga Guajardo (Faculty of Medicine, Universidad Autónoma de Nuevo Leon, Monterrey, N.L., Mexico), Maria Elena Cedano Limon (private practice, Mexico City, Mexico), Roberto Bejarano Rodriguez (private practice, Mexico City, Mexico), Lirio de Maria Delgado Garcia (private practice, Chihuahua, Chih., Mexico), Juan Carlos Villanueva Arias (private practice, Guadalajara, Jal. Mexico), Lucia Alejandra Castillo Vigna (private practice, Guadalajara, Jal. Mexico), José Gerardo González González (Faculty of Medicine, Universidad Autónoma de Nuevo Leon, Monterrey, N.L., Mexico), and Martha Leticia López Velazco (private practice, Guadalajara, Jal. Mexico). The authors apologize for this omission. 